\title{
Atrioventricular Node Dysfunction in Adult Patient with Atrial Septal Defect: Focus on Pacemaker Management
}

\author{
Muhammad Ulil Aidie Jomansyah, Dyah Wulan Anggrahini, Erika Maharani \\ Department of Cardiology and Vascular Medicine, Faculty of Medicine, Universitas Gadjah Mada - \\ Dr. Sardjito Hospital, Yogyakarta, Indonesia \\ *correspondence: ulilaidie@gmail.com
}

\begin{abstract}
Introduction: Bradycardia is a condition when the heart rhythm below 60 times per minute. This condition can caused by sinus node dysfunction or atrioventricular node dysfunction. SND and/ or AVND often accompany with congenital heart disease and make a worst patient condition. Pacemaker implantation is importance to prevent heart failure condition or sudden cardiac death in this condition. The importance of pacemaker implantation preparation and technique in this setting underlies this case report presentation.

Case: A 33 years old male, reffered from private hospital with chief complain recurrent near syncope since 5 month ago. Patient also felt easy to fatique while activity, chest pain and palpitation. Patient did not complain about leg swelling, bluish finger and lips. Patient and his family has not history about cardiac disease. On examination, he was fully alert and healthy appearing, with blood pressure 130/80 $\mathrm{mmHg}$, heart rate 62 beat per minute and reguler, respiratory rate 18 times per minute, and temperature $36.5 \mathrm{C}$. Heart examination showed RV heaving, S1 normal and S2 fixed wide split, systolic murmur 2/6 at left linea parasternal SIC IV and no sign of cardiomegaly. His electrocardiogram showed AV block grade I with normal axis and no sign of RVH (figure 1). Echocardiography result was secundum atrial septal defect left to right shunt, normal ejection fraction and mild tricuspid regurgitation (figure 2). Holter monitoring showed SA exit block and sustained supraventricular tachycardia, AV block grade I to high degree AV block and periodic ventricular standstill(figure 3).

Discussion: Sinus Node Dysfunction (SND) and/or Atrioventricular Node Dysfunction (AVND) are the primary spectrum of bradycardia that could reduce the heart rate and promote heart failure condition and sudden cardiac death, especially in congenital heart disease (CHD) patient. These condition could occur as a congenital process or as a complication of CHD correction. The patient with atrial septal defect who had a episode of bradycardia symptomatic and proved by holter monitoring,the urgent pacemaker implantation is important to minimalizing the risk of worsening condition andsudden cardiac death. Patient can undergo the correction of ASD followed by implantation of dual chamber endocardial permanent pacemaker in the same time. But, if the ASD condition is not viable to be corrected, the endocardial pacemaker is not recommended to be performed due to high risk of thromboembolism complication. Epicardial permanent pacemaker is the best optional technique to implant the pacemaker in this condition.

Conclusion: SND and/or AVND is a bad comorbid in atrial septal defect patient that can lead to worse heart failure condition and sudden cardiac death. The urgent total correction of ASD and followed by implantation of dual chamber endocardial permanent pacemaker is the best choice management in this patient.
\end{abstract}

